\title{
Identification of possible compounds possessing adenosine A1 receptor binding activity in the leaves of orthosiphon stamineus using TLC and multivariate data analysis
}

\begin{abstract}
A novel approach to identify compounds possessing adenosine A1 receptor binding activity in the leaves of $\mathrm{O}$. stamineus was developed. O. stamineus extract is one of the components of a functional beverage used in Indonesia for the treatment of kidney stones. In this study, adenosine A1 receptor binding, which is related to the diuretic action in the treatment of kidney stones was tested. A combination of thin layer chromatography of different extracts prepared by extraction with diverse solvents (n-hexane, chloroform, n-butanol and water), and multivariate data analysis based on orthogonal partial least squares proved to be a promising approach to determine these active compounds. Several methoxyflavonoids, fatty acids or terpenoids were estimated to be related to this activity. The results of this study support the traditional use in Indonesia of $\mathrm{O}$. stamineus as a functional drink to treat kidney stones.
\end{abstract}

Keyword: Adenosine; Kidney stone; Multivariate data analysis; Orthogonal partial least square; Orthosiphon staminues; TLC 